\title{
Design of Fluorescent Bentonite/Carbon Dots Nanomaterial for Lead Removal
}

\author{
Khouloud Jlassi ${ }^{1^{*}}$, Abdelgalil Khalaf Ahmed ${ }^{2}$, Doaa Fadhel Farhan ${ }^{2}$, \\ Aboubakr M. Abdullah ${ }^{1^{*}}$, Mohamed M. Chehimi ${ }^{3^{*}}$, Igor Krupa ${ }^{1}$
}

1. Center for Advanced Materials, Qatar University, P.O. Box 2713, Doha, Qatar

2. College of Arts and Sciences, Qatar University, P.O. Box 2713, Doha, Qatar

3. Univ Paris Est Creteil, CNRS, ICMPE, UMR7182, F-94320 Thiais, France

\begin{abstract}
${ }^{*}$ Correspondence:
khouloud.jlassi@qu.edu.qa (KJ); abubakr2@yahoo.com (AMA); chehimi@icmpe.cnrs.fr and mmchehimi@yahoo.fr (MMC)
\end{abstract}

\section{Abstract}

This work describes the preparation of novel fluorescent bentonite clay (BP), modified with carbon quantum dots nanomaterials (CDs), and its usage as lead removal platform, the CDs was prepared using graphitic waste serving as carbon source material via hydrothermal method, and the as obtained CDs were found to be fluorescent, in spherical shape, positively charged and smaller than $5 \mathrm{~nm}$. Encouraged by their structure and photoluminescence feature, they were used hereafter as intercalants or surface modifiers, in order to make fluorescent bentonite nanocomposites. Bentonite was used as negatively charged model of aluminosilicate and reacted with the positively charged CDs. Interestingly, CDs were found to intercalate bentonite as judged from XRD patterns, and TEM. Indeed, the basal distance of bentonite clay $\mathrm{d}(001)$ shifted from 1.2 $\mathrm{nm}$ to $2.9 \mathrm{~nm}$, after bentonite modification using the prepared carbon dots; moreover the XRD pattern of BP-CDs recorded in the some regions show some additional diffraction peaks along with those for bentonite. The peaks centered at $2 \theta=27$ degree allocated to the facets of graphitic-like carbon, originated from the introduced carbon dots inside bentonite galleries. The prepared materials were characterized by XPS, FTIR and fluorescence analysis. The obtained results indicate that CDs were successfully intercalated inside bentonite matrix and found to be stable over time. The BP-CD nanocomposites were finally used as efficient hybrid platform for led removal with and extraction efficiently of $95 \%$ under light condition, room temperature under alkaline conditions and after only $10 \mathrm{~min}$ of reaction.

\section{Keywords:}

Carbon quantum dots, Bentonite, Hydrothermal process, heavy metal removal 


\section{Introduction}

Bentonite is a layered aluminosilicate of substantial interest among 2D nanomaterials due to its abundance, low cost, high surface area, layered structure, anionic nature with excellent cation exchange capacity, and chemical reactivity (e.g. silanization, arylation). Bentonite is employed in a variety of emergent domains and applications, namely in catalysis, water treatment for heavy metal removal and energy storage. Although bentonite has appealing features, one could take advantage of its reactivity to finely tune its final properties whether it is considered alone or in bentonite-based composites. For example, bentonite clays could be modified using using, polymers, nucleic acids, surfactants, silanes and diazonium salts in order to turn it organophilic and to tailor interfaces/interphases in bentonite-based composites with a result of outstanding performances (e.g. selective adsorption, sensing, mechanical reinforcement) (Jlassi et al. ISBN: 9780323461535). Of relevance to the present work, bentonite clay was modified with carbon nanomaterials such as graphite (https://doi.org/10.1166/mex.2020.1630 ), graphene (https://doi.org/10.1007/s11356-020-09423-7 ) and multi-walled carbon nanotubes (https://doi.org/10.1177/0021998319848798 ); the resultant hybrid materials were widely used in renewable energy, electronics and environment remediation applications. The resulting hybrids combine both high surface area, adsorptive performance of the 2D nanoclays and high active sites, electric performance of carbon nanomaterials; this synergetic combination was expected to promote the performance of the targeted composites, especially in environmental domain. To the best of our knowledge, no study has been directed towards the preparation of natural clay intercalated with carbon quantum dots. This is an important step in the design layered aluminosilicate/carbon nanomaterial as carbon quantum dots have the unique feature to be fluorescent, which is not the case of CNTs and graphene.

Herein, we report a novel and easy way to prepare a fluorescent and hybrid, bentonite-modified with carbon quantum dots via hydrothermal process, the latter was prepared from graphitic waste, and the bentonite served as Hosting matrix, to support, avoid the agglomeration and preserve the fluorescent properties of the as prepared carbon quantum dots. The resulting material is tested as hybrid platform for lead removal. 


\section{Materials and Methods}

\subsection{Chemicals and materials}

Graphite wastes were collected from Qatalum (Doha, Qatar). $\mathrm{H}_{2} \mathrm{SO}_{4}, \mathrm{HNO}_{3}$, ammonia and bentonite nanoclay were purchased from Sigma-Aldrich (Munich, Germany).

\subsection{Synthesis of CDs, B-CDs}

In order to obtain nitrogen dopped carbon quatum dots (CDs), $4 \mathrm{~g}$ of graphite waste was initially grinded then dissolved in sulfuric and nitric acids under stirring/refluxing, as previously described[1], the as obtained CDs was introduced to bentonite clay(BP) suspension, in order to obtain the (BP-CDs )nanocomposite through cation exchange reaction, 1g of BP (negatively charged) was mixed with 20 wt.\% of CDs (positively charged), the mixture was kept under mechanical stirring at room temperature for $24 \mathrm{~h}$, centrifuged, washed and dried at $60 \mathrm{C}$. The as prepared material (B-CDs) was used hereafter as hybrid platform for heavy metal removal, heater for led removal.

\subsection{Adsorption experiment}

The adsorption of BP and BP-CDs was followed by using batch technique. Adsorption processes for single-factor experiments were using $0.6 \mathrm{~g}$ of BP and BP-CDs nanomaterials and $50 \mathrm{~mL}$ of led solution ( $5 \mathrm{ppm})$, placed on an orbital shaker at $180 \mathrm{rpm}$ and shaken between five and $45 \mathrm{~min}$ until the equilibrium was reached.

\subsection{Characterizations}

The $\mathrm{BP}, \mathrm{CDs}$ and $\mathrm{B}-\mathrm{CDs}$ prepared materials were analyzed using transmission electron microscope (TEM, TecnaiG220, FEl, Hillsboro, OR, USA), X-ray photoelectron spectroscopy (XPS) measurements were conducted using a Kratos Shimadzu Axis Ultra DLD machine (Kratos, Manchester, UK) equipped with a monochromatic Al Ka radiation source (1486.6 eV) under a UHV environment (ca. 5x10-9 Torr). The X-ray diffraction pattern (XRD) was investigated on an X-ray diffractometer (X'Pert-Pro MPD, PANalytical Co., Almelo, Netherlands) using Cu Ka X-ray source $(\lambda=1.540598 \AA$ ) . The Fourier transform infrared spectra were characterized on a Thermo Nicolet Nexus 670 FTIR spectrometer (Thermo Scientific, Madison, WI, USA), and to investigate the optical properties, sample characterization was carried out on a Shimadzu UV-3600 spectrophotometer, FLS920 P fluorescence spectrometer (Edinburgh Instruments). 


\section{Results and discussion}

\subsection{Preparation of CDs and B-CDs}

The preparation of new Carbon-dots (CDs) from graphitic waste, via hydrothermal treatment in the presence of ammonia. This process yields outstanding photo luminescent, and monodispersed carbon dots with an average size smaller than $5 \mathrm{~nm}$ fig $1(\mathrm{a}, \mathrm{b})$. The as obtained CDs are nitrogen doped, and exhibit unprecedented photo luminescent properties. CDs were used to make fluorescent bentonite clay, via hydrothermal process fig $1(b, c)$; the bentonite clay was used hereafter as hybrid platform for heavy metal removal. We have used powerful techniques to characterize these unique dots and their hybrids (Fig.4).

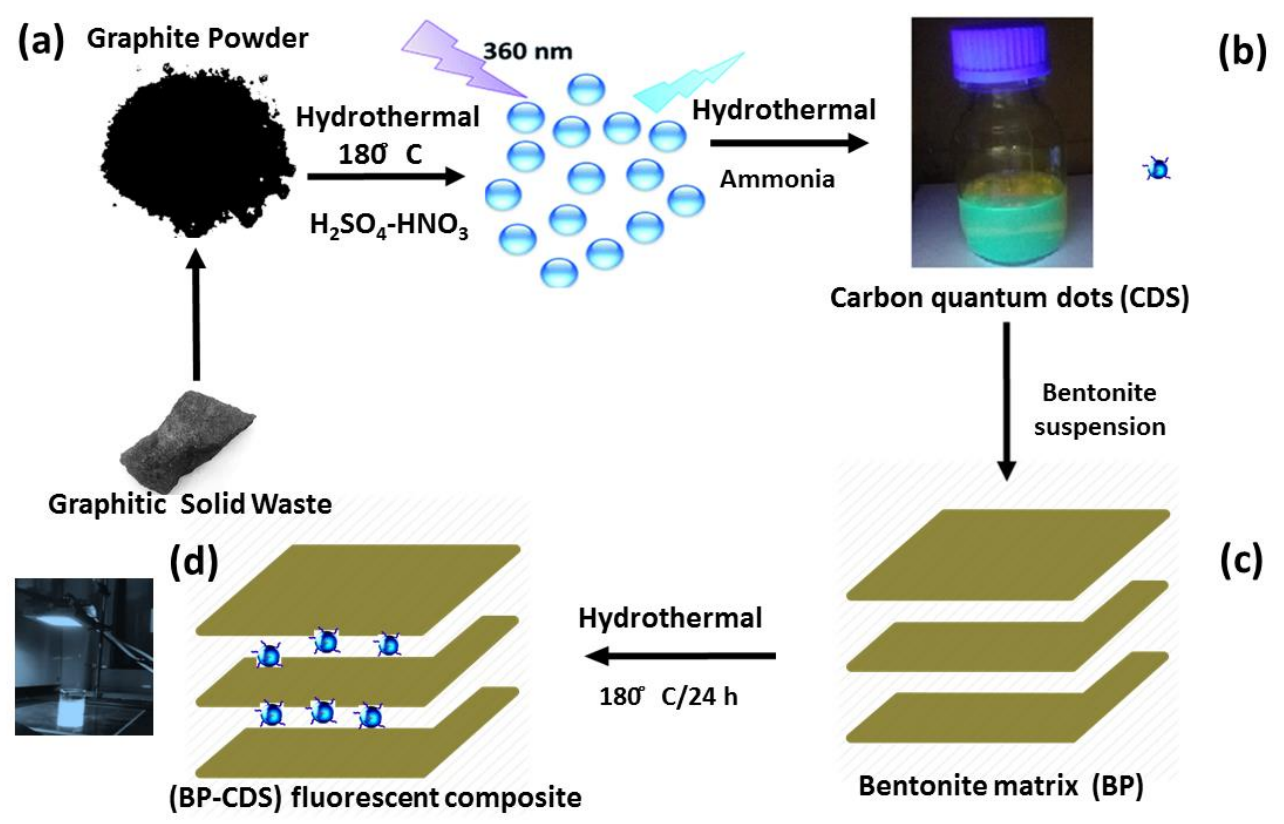

Fig.1. Upper panel (a) and (b) showing the sequential steps of CDs preparation, lower panel (d) and (c) showing the surface modification of bentonite using the fluorescent CDs via hydrothermal process, in order to obtain fluorescent bentonite (B-CDs).

The TEM images (Fig.2a) revealed spherical and well dispersed nitrogen doped CDs, with an average diameter of $5 \mathrm{~nm}$. From the corresponding X-ray diffraction (XRD) pattern of $\mathrm{B}$ and $\mathrm{B}$ CDs (Fig.2d) and applying the Bragg equation, the basal distance of pure bentonite was found to be equal to $1.3 \mathrm{~nm}$, after cation exchange reaction with the negatively charged CDs, the basal distance was found to increase up to $2.9 \mathrm{~nm}$, indicating a successful cation exchange reaction, 
between the positively charged clay and prepared CDs, The interlayer distance of BP and B-CDs was investigated as well using TEM images $\mathrm{m}$, and the average distance was calculated trough 2 selected regions, and was found on the same range than the distances deduced from XRD (Fig.2b and c). XPS was employed to investigate the electronic configuration and surface composition $\mathrm{BP}, \mathrm{CDs}$ and $\mathrm{BP}-\mathrm{CDs}$, as the survey spectra permit to pathway the most important changes in the surface composition of CDs and BP surface after and before modification, BP-CDs exhibit intense 01s peak, N 1s and C1s (Fig. 3a).

\subsection{Physical structure}

Fig. 2 shows TEM and XRD patterns of CDs, BP and BP-CDs. Fig 2.a shows a spherical CDs with an average size smaller than $5 \mathrm{~nm}$, as previously reported [2]. Fig 2.b shows the layered structure of bentonite clay, the basal distance from three selected regions equals to $\sim 1.2 \mathrm{~nm}$, fig $2 \mathrm{c}$ shows the intercalated clay layers in the presence of well dispersed carbon dots; the average basal distance of bentonite clay was found to be equal to $2.8 \mathrm{~nm}$.
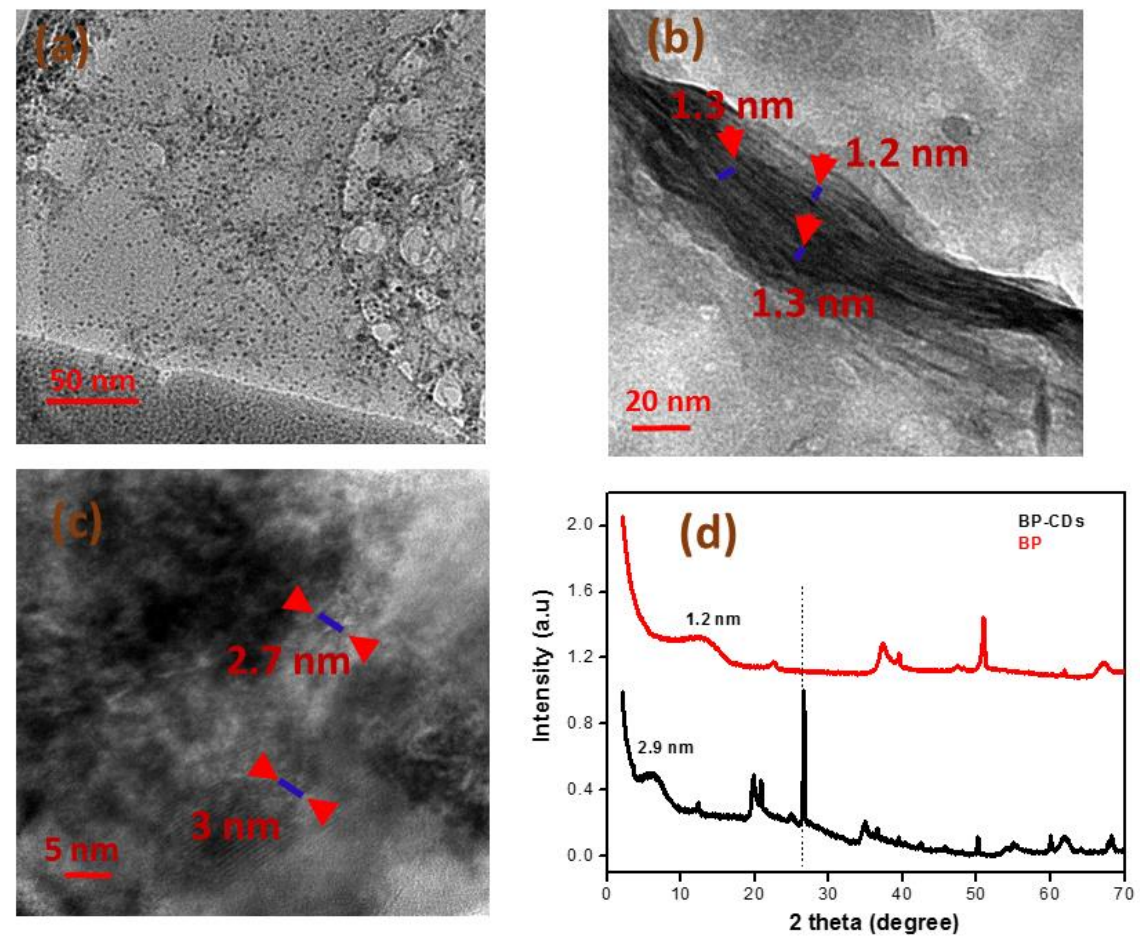

Fig.2. (a), (b) and (c) TEM of BP, CDs and BP-CDs at different magnifications, (d) XRD patterns of $\mathrm{BP}$ and $\mathrm{B}-\mathrm{CD}$ s nanohybrids. 
XRD patterns of BP, CDs and BP-CDs are shown in Fig.2d. The typical diffraction peaks related to bentonite shift to smaller angles after the modification with $\mathrm{CDs}$, as concluded from Bragg equation. The basal distance of bentonite clay shifted from 1.2 to $2.9 \mathrm{~nm}$ for BP-CDs respectively, in the same range of the basal distances concluded from TEM images, whereas XRD pattern of BP-CDs recorded in the some regions show some additional diffraction peaks along with those for bentonite. The peaks centered at $2 \theta=27^{\circ}$ allocated to the facets of graphitic-like carbon. These TEM and XRD results account for the intercalation of CDs into the bentonite matrix.

\subsection{Chemical structure}

XPS analysis was conducted in order to investigate, surface composition of BP-CD nanocomposite and reference materials. Figure 3 displays survey spectra of B-CDs and CDs which confirm the presence of more intense $\mathrm{C}$ 1s and N1s peaks for the BP-Cd nanocomposite indicating modification of the clay by CDs.

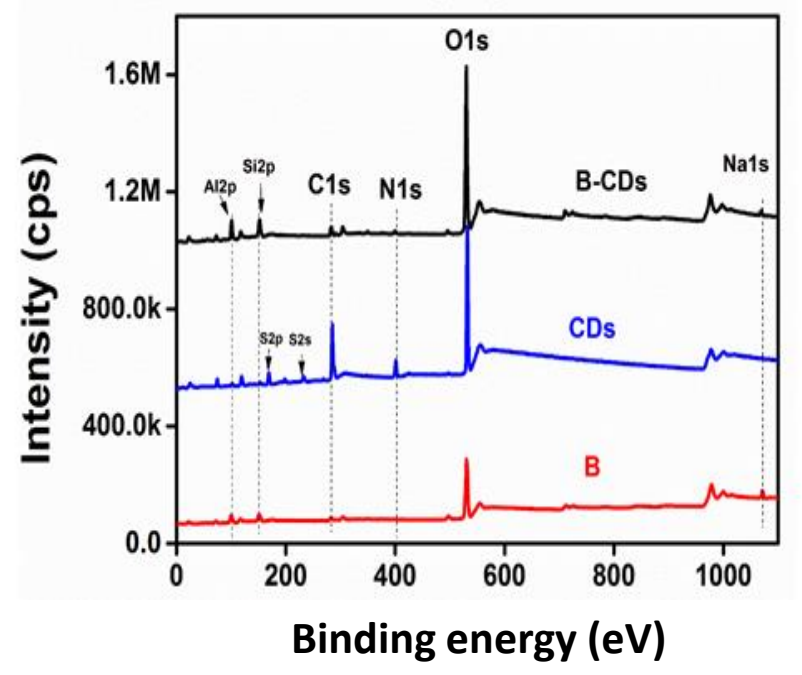

Fig.3. XPS survey spectra and bentonite, CDs and their nanocomposite BP-CD. 
Table 1. XPS-determined apparent surface composition of BP. CDs and BP-CDs.

\begin{tabular}{|l|l|l|l|l|l|l|l|}
\hline Materials & $\mathrm{Si}$ & $\mathrm{Al}$ & $\mathrm{O}$ & $\mathrm{Na}$ & $\mathrm{C}$ & $\mathrm{N}$ & $\mathrm{S}$ \\
\hline $\mathrm{BP}$ & 19.5 & 8.30 & 64.6 & 0.72 & 6.95 & - & - \\
\hline $\mathrm{CDs}$ & 1.5 & 8 & 39.9 & - & 39.4 & 7.3 & 3.9 \\
\hline BP-CDs & 15.65 & 7.18 & 65 & 0.12 & 10.5 & 1.7 & \\
\hline
\end{tabular}

The chemical compositions of the prepared nanomaterials were reported in Table 1. The atomic ratios $\mathrm{C} / \mathrm{N} / \mathrm{O}$ were determined to be $10.5 / 1.7 / 65$ for $\mathrm{BP}-\mathrm{CDs}$, whereas for $\mathrm{CDs}$ and $\mathrm{BP}$ the ratio is equal to $39.4 / 7.3 / 39.9$ and $6.95 / 0 / 64.6$, respectively. The detection of nitrogen indicates is due to doping of the prepared CDs, which implies that bentonite is loaded with $\mathrm{N}$-doped $\mathrm{CD}$ as judged from the surface elemental composition of BP-CD. The decrease in the atomic percent of sodium on going from BP to BP-CD is in line with modification of the clay by intercalation of the quantum dots.

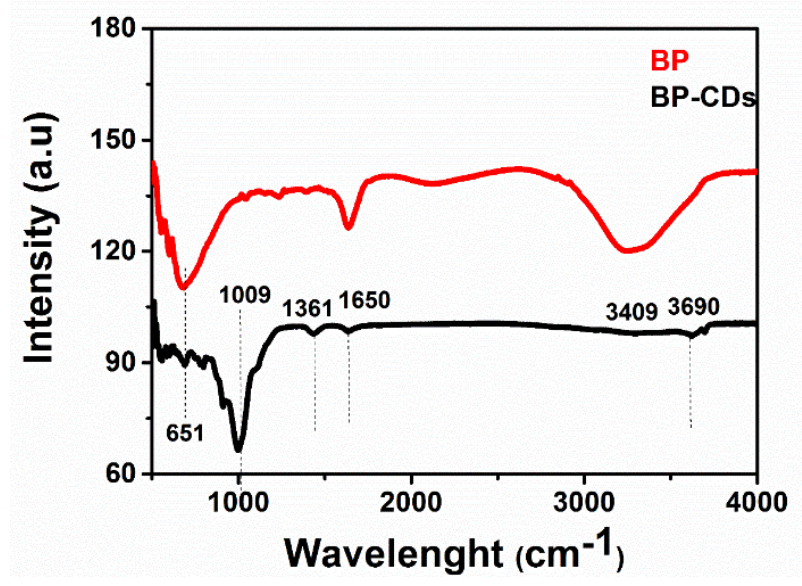

Fig. 4-FTIR spectra of BP and BP-CDs

FTIR spectra are shown in Fig.4 for bentonite before and after modification with CDs. It is important to note the decrease of $\mathrm{OH}$ bands intensity, situated at 3413 and $1637 \mathrm{~cm}^{-1}$ reflecting the intercalation of the CDs with the clay surface. Moreover, peaks situated at 651 and 1009 $\mathrm{cm}^{-1}$, could be associated with Si-O-Si, Mg-O-Si vibrations[3, 4], the peak at $1361 \mathrm{~cm}^{-1}$ for BP- 
CDs material is associated with C-O vibration bonds, and the one at $1457 \mathrm{~cm}^{-1}$ is associated C-N bonds, Moreover the presence $\mathrm{C}=\mathrm{C} / \mathrm{C}=\mathrm{O}$ bonds could be detected due to the band situation $1650 \mathrm{~cm}^{-1}, 3409 \mathrm{~cm}^{-1}$ band reveals the presence of $\mathrm{O}-\mathrm{H}$ and $\mathrm{N}-\mathrm{H}$ groups in BP-CDs material[5].

\subsection{Optical properties of CDs}

The UV-Vis absorption of CDs (Fig. 5a), displays two absorption peak centered at 380 and 450 $\mathrm{nm}$, assigned to $\pi \rightarrow \pi^{*}$ transition and $\mathrm{n} \rightarrow \pi^{*}$ transition, similar to previously reported carbon quantum dots-based materials[1,6]. The emission spectra (Fig. 5.C) of the CDs show the increase in the PL intensity as the excitation wavelength is increasing from 300 to $460 \mathrm{~nm}$. The maximum emission intensity reached $454 \mathrm{~nm}$ (blue emission) when it is excited at $460 \mathrm{~nm}$. This phenomena is associated with carbon quantum dots [7].
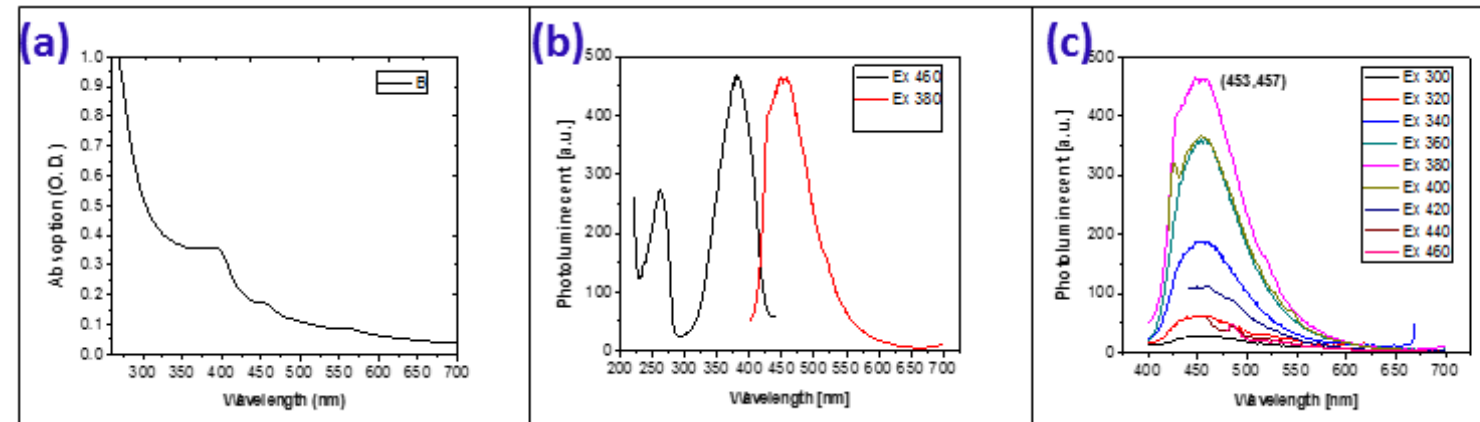

Fig.5. (a) UV-Vis absorption spectrum, (b) PLE absorption spectrum (c) excitation-dependent emission spectra of CDs at different excitation wavelengths.

\subsection{Removal of $\mathrm{Pb}^{2+}$ in water using $\mathrm{BP}$ and $\mathrm{BP}-\mathrm{CD}$ hybrids}

The adsorption performance of $\mathrm{Pb}^{2+}$, using $\mathrm{BP}$ and (BP-CDs) nanohybrids was studied, as well as

the $\mathrm{pH}$ and contact time effect. $\mathrm{BP}$ and $\mathrm{BP}-\mathrm{CD}$ s were immersed in a 5-ppm solution of $\mathrm{Pb}^{2+}$ up to $45 \mathrm{~min}$, the remaining concentration of $\mathrm{Pb}^{2+}$ was detected using (ICP) analysis technique, as shown in fig (6a), the extraction efficiency percentage of $\mathrm{Pb}^{2+}$ was found to increase in alkaline solution, the highest extraction efficiency percentages $(E=95 \%)$ were noted in alkaline solution a $\mathrm{pH}=8$ at room temperature, using $\mathrm{BP}-\mathrm{CDs}$ monohybrids compared to $81 \%$ only in presence of unmodified BP. 

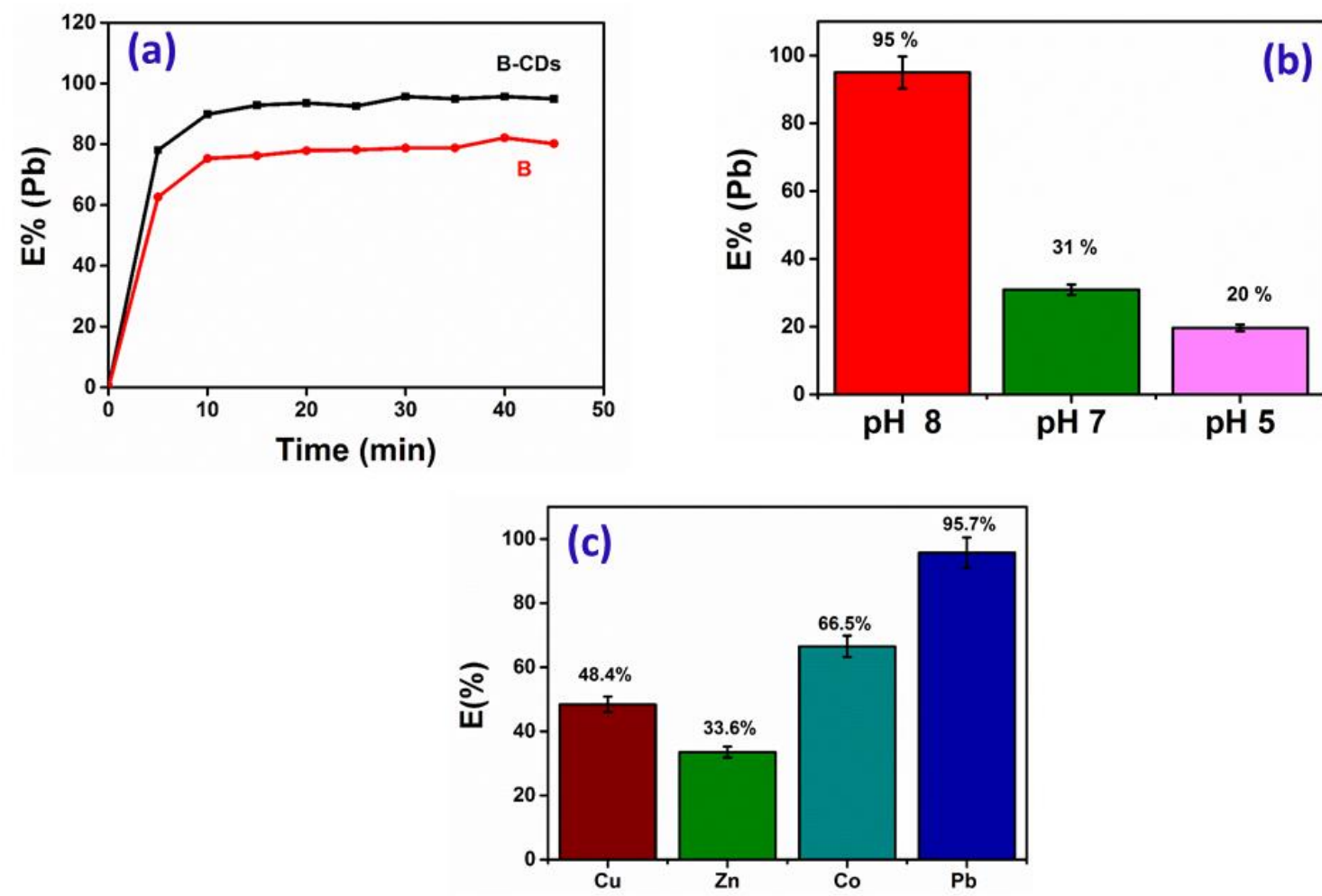

Fig.6. (a) Trends of the extraction efficiency percentages for cadmium using BP, BP-CDs under light at $\mathrm{pH}=8$, (b) Trends of the extraction efficiency percentages for lead at different $\mathrm{pH},(\mathrm{c})$ selectivity test.

Fig.6a show the trends of extraction efficiency percentages at different time in presence of 5 ppm of lead using BP, and BP-CDs under light at $\mathrm{pH}=8$, as shown after 10 minutes only The BPCDs sample reached the maximum extraction efficiency(95\%) under light, while the BP reached $81 \%$.

\section{Conclusion}

In conclusion, BP-CDs were successfully prepared via hydrothermal process using CDs from graphitic waste; natural bentonite served as hosting matrix. The CDs were successfully introduced into bentonite nanoclay matrix and form well dispersed BP-CDs, stable and fluorescent. The latter was successfully used as platform for lead removal from water, with and extraction efficiency around 95\%. The equilibrium time of adsorption was faster than other reported works (only $10 \mathrm{~min}$ ). In addition, the optimal pH of the solution was 8 . 


\section{Acknowledgement:}

This report was made possible by a UREP award [UREP25-091-2-034] from the Qatar National Research Fund (a member of The Qatar Foundation). The statements made herein are solely the responsibility of the authors.

\section{References}

[1] K. Jlassi, K. Eid, M.H. Sliem, A.M. Abdullah, M.M. Chehimi, I. Krupa, Rational synthesis, characterization, and application of environmentally friendly (polymer-carbon dot) hybrid composite film for fast and efficient UV-assisted Cd 2+ removal from water, Environmental Sciences Europe, 32(2020) 1-13.

[2] K. Jlassi, S. Mallick, A. Eribi, M.M. Chehimi, Z. Ahmad, F. Touati, et al., Facile preparation of NS co-doped graphene quantum dots (GQDs) from graphite waste for efficient humidity sensing, Sensors and Actuators B: Chemical, 328129058.

[3] M. Mudasir, R.A. Baskara, A. Suratman, K.S. Yunita, R. Perdana, W. Puspitasari, Simultaneous adsorption of $\mathrm{Zn}$ (II) and $\mathrm{Hg}$ (II) ions on selective adsorbent of dithizone-immobilized bentonite in the presence of Mg (II) Ion, Journal of Environmental Chemical Engineering, 8(2020) 104002.

[4] K. Jlassi, K. Eid, M.H. Sliem, A.M. Abdullah, M.M. Chehimi, Calix [4] arene-clicked clay through thiol-yne addition for the molecular recognition and removal of $\mathrm{Cd}$ (II) from wastewater, Separation and Purification Technology, 251(2020) 117383.

[5] K. Dimos, F. Arcudi, A. Kouloumpis, I.B. Koutselas, P. Rudolf, D. Gournis, et al., Top-down and bottom-up approaches to transparent, flexible and luminescent nitrogen-doped carbon nanodot-clay hybrid films, Nanoscale, 9(2017) 10256-62.

[6] Y. Liu, P. Wu, X. Wu, C. Ma, S. Luo, M. Xu, et al., Nitrogen and copper (II) co-doped carbon dots for applications in ascorbic acid determination by non-oxidation reduction strategy and cellular imaging, Talanta, 210(2020) 120649.

[7] Y. Yang, D. Wu, S. Han, P. Hu, R. Liu, Bottom-up fabrication of photoluminescent carbon dots with uniform morphology via a soft-hard template approach, Chemical Communications, 49(2013) 4920-2. 